\title{
TENSIONES DE CRECIMIENTO LONGITUDINALES EN ÁRBOLES DE Eucalyptus nitens CON DIMENSIONES ASERRABLES
}

\section{LONGITUDINAL GROWTH STRESSES IN Eucalyptus nitens TREES WITH SAWABLE DIMENSIONS}

\author{
Margarette Omonte ${ }^{1, \wedge}$, Luis Valenzuela
}

\begin{abstract}
RESUMEN
En el presente estudio se investigaron los niveles de tensiones de crecimiento longitudinales en árboles de Eucalyptus nitens con dimensiones aserrables. Junto con esto, se investigó la variación fustal de las tensiones de crecimiento longitudinales, del indicador de la tensión de crecimiento y del módulo de elasticidad de la madera en tensión, y se examinan las relaciones entre estas variables. Se escogieron aleatoriamente nueve árboles de una plantación de Eucalyptus nitens de 18 años de edad, crecida en la Región del Biobío, Chile. En cada árbol se midió el indicador de la tensión de crecimiento, en las exposiciones Norte, Sur, Este y Oeste, para una altura fija de 2 m, y al 33, 66 y 100\% de la altura comercial del árbol, así como también, se determinó el módulo de elasticidad de la madera en tensión y las tensiones de crecimiento longitudinales en estas mismas exposiciones y alturas. El nivel medio de la tensiones de crecimiento longitudinales encontrado fue de 28,3 MPa. Las tensiones de crecimiento longitudinales, el indicador de la tensión de crecimiento y el módulo de elasticidad de la madera en tensión, variaron significativamente a través de las alturas y exposiciones en el fuste. Se encontró una alta influencia del indicador de la tensión de crecimiento en la estimación de los niveles de tensiones de crecimiento longitudinales.
\end{abstract}

Palabras claves: Deformación de crecimiento, módulo de elasticidad, módulo de rigidez, propiedades de la madera, variabilidad de la madera.

\begin{abstract}
In this study, the levels of longitudinal growth stresses in Eucalyptus nitens trees with sawable dimensions were investigated. Furthermore, tree stem variations of the longitudinal growth stresses, growth stress indicator and modulus of elasticity in tension were also investigated, and the relationships between these variables were examined. Nine trees were randomly selected from a plantation of Eucalyptus nitens trees of 18 years old, grown at the Biobío region, Chile. Growth stress indicator were measured in each tree at North, South, East and West stem expositions, at $2 \mathrm{~m}$ height, and at 33, 66 and $100 \%$ of the tree merchantable height, in which modulus of elasticity in tension and longitudinal growth stresses were also determinated. The mean level of longitudinal growth stresses found was 28,3 MPa. All variables (longitudinal growth stresses, growth stress indicator and modulus of elasticity in tension) showed high variability through the expositions and heights in the tree stem. High influence of growth stress indicator on the estimation of longitudinal growth stresses was found.
\end{abstract}

Keywords: Growth strain, modulus of elasticity, stiffness, wood properties, wood variability

\footnotetext{
Laboratorio Silvotecnológico de la Madera, Facultad de Ciencias Forestales, Universidad de Concepción, Concepción ,Chile.

^Autor para correspondencia: margaretomonte@udec.cl

Recibido: 08.07.2014 Aceptado: 21.03.2015
} 


\section{INTRODUCCIÓN}

Un factor clave que limita el uso de las plantaciones del género Eucalyptus, como madera aserrada de calidad, son los altos niveles de tensiones de crecimiento en el interior de la madera (Yang et al. 2001, Yang y Waugh 2001, Yang et al. 2005, Trugilho et al. 2006). Estas tensiones, que se encuentran en equilibrio en el árbol en pié (Malan y Gerischer 1987), son liberadas durante la corta y trozado del árbol, y a lo largo de las operaciones de aserrío posteriores, generando un conjunto de deformaciones que provocan distintos defectos en las trozas o productos ya aserrados (Touza 2001, INFOR 2004).

Las tensiones de crecimiento son originadas durante el proceso de maduración de las células que produce el cambium (Nicholson 1973, Trugilho et al. 2002). Durante este proceso, las células que crecen cada año sobre la periferia del fuste, tienden a expandirse lateralmente y a contraerse longitudinalmente, sin embargo, estos cambios dimensionales son impedidos por las células que ya están lignificadas y que han sido formadas en años anteriores, lo que genera un conjunto de tensiones (Vignote et al. 1996, Touza 2001, Yang y Waugh 2001, Yang et al. 2005, Biechele et al. 2009). Con el paso del tiempo estas tensiones se acumulan, y como consecuencia, el centro del fuste se encuentra sometido a tensiones de compresión, cuya magnitud aumenta hacia la médula, mientras que la periferia del tronco, se encuentra sometida a esfuerzos en tracción que se incrementan en dirección a la corteza (Touza 2001, Yang et al. 2005).

Las tensiones de crecimiento en dirección longitudinal son las más intensas y variables (Trugilho y Oliveira 2008), y las más importantes desde el punto de vista de la calidad de la madera sólida (Yang y Waugh 2001). Aunque las tensiones de crecimiento no pueden ser medidas directamente, estas pueden ser calculadas a partir de mediciones de deformación y del módulo de elasticidad de la madera en tensión, $\boldsymbol{E}_{T}$ (Yang y Waugh 2001, Raymond et al. 2004, Yang et al. 2005).

La deformación en dirección longitudinal, que es provocada por la liberación de las tensiones de crecimiento longitudinales $\left(\sigma_{L}\right)$, es considerada como un estimador directo de los niveles de $\sigma_{L}$ (Valencia et al. 2011). Distintos métodos han sido desarrollados para poder medir estas deformaciones longitudinales, siendo los más utilizados: el método Nicholson, el método CIRAD-Forêt y el método "strain gauge"; y que básicamente difieren entre sí, en la forma en que las $\sigma_{L}$ son liberadas, y por lo tanto, en la cantidad de deformación longitudinal que es medida (Yang y Waugh 2001).

El método CIRAD-Fôret permite realizar mediciones repetibles de deformación longitudinal (Raymond et al. 2004) y puede ser considerado como un método no destructivo debido a su reducido daño físico en el fuste (Trugilho y Oliveira 2008). Este método básicamente permite registrar el desplazamiento longitudinal que experimentan dos clavos insertos en la madera a una distancia fija, luego de liberar las $\sigma_{L}$. Este desplazamiento registrado, es conocido como "indicador de la tensión de crecimiento", GSI, y aunque no corresponde directamente a la deformación longitudinal, se considera proporcional a ésta (Yang et al. 2005), y por lo mismo han sido ampliamente utilizados para estimar los niveles de $\sigma_{L}$ (Raymond et al. 2004, Valdés 2004, Vignote et al. 1996, Biechele et al. 2009, Medhurst et al. 2011, Valencia et al. 2011, Díaz et al. 2012).

La deformación longitudinal puede ser obtenida a través del GSI, y mediante un factor de conversión que depende la geometría del sensor y de la anisotropía elástica de la madera en el plano longitudinal-tangencial (Baillères 1994, Fournier et al. 1994, Yang et al. 2005).

La utilización de las especies del género Eucalyptus en usos de mayor valor agregado, se ve limitada por la presencia de altos niveles de $\sigma_{L}$ en la madera (Yang et al. 2001, Yang y Waugh 2001, Yang et al. 2005, Trugilho et al. 2006). En Chile, Eucalyptus nitens (Deane \& Maiden) es la especie forestal de más rápido crecimiento que ha sido establecida industrialmente (INFOR 2004, Valencia y Cabrera 2009). Sin embargo, y aunque esta especie ha alcanzado un alto grado de desarrollo y aceptación como materia prima para la fabricación de celulosa, estudios sobre la calidad de su madera para generar productos sólidos son aún escasos (Díaz et al. 2012). 
La mayoría de los estudios en Eucalyptus nitens en relación a las $\sigma_{L}$, miden el GSI a través del método CIRAD-Fôret, y lo utilizan como un indicador del nivel de $\sigma_{L}$ (Valdés 2004, Biechele et al. 2009, Medhurst et al. 2011, Valencia et al. 2011, Díaz et al. 2012). Trabajos en los cuales se calculan las $\sigma_{L}$, sólo han sido realizados en árboles jóvenes, de 8 años de edad (Chafe 1985, 1990).

A pesar de los distintos efectos no deseados que pueden provocar los altos niveles de $\sigma_{L}$, en la utilización de la madera sólida, descritos con detalle por Yang y Waugh (2001), y de la importancia que tiene el conocimiento de la distribución fustal de estas tensiones para la generación de estrategias de aserrío adecuadas, no existe información acerca de los valores que estas $\sigma_{L}$ pueden alcanzar en árboles de Eucalyptus nitens con dimensiones aserrables, ni tampoco de su variación a lo largo del fuste.

En el presente estudio se investigó el nivel de $\sigma_{L}$ en árboles de Eucalyptus nitens con dimensiones aserrables, de 18 años de edad. Junto con esto, se investigó la variación fustal de las $\sigma_{L}$, del $G S I$, y del $E_{T}, \mathrm{y}$ además, se examinaron las relaciones entre éstas variables.

\section{MATERIALES Y MÉTODOS}

El estudio fue realizado en una plantación de Eucalyptus nitens de 18 años de edad, ubicada a 340 m s.n.m. en la Región del Biobío, Chile ( $37^{\circ} 51^{\prime} \mathrm{S}$ y $\left.72^{\circ} 07^{\prime} \mathrm{O}\right)$. La plantación fue manejada para producir madera sólida y fue intervenida con dos raleos, efectuados a los 7 y 9 años de edad, con densidades residuales de 500 y 300 árboles ha $^{-1}$, respectivamente. El sitio de la plantación presenta una topografía plana y pendientes inferiores a $7 \%$, una precipitación promedio anual que supera los $1000 \mathrm{~mm}$, distribuidos en un $80 \%$ entre abril y octubre, y temperaturas promedios que oscilan entre los $5^{\circ} \mathrm{C}$ la mínima y los $21^{\circ} \mathrm{C}$ la máxima (Díaz et al. 2012).

Se escogieron nueve árboles aleatoriamente, sin evidencia de daño o enfermedad, y sin inclinación con respecto a la posición vertical. Con un diámetro a $1,3 \mathrm{~m}$ sobre el nivel del suelo $(D A P)$ mínimo y máximo de 28,8 y $48,1 \mathrm{~cm}$ respectivamente, y con alturas totales en un rango de 39,3 a 44,7 m.

Para analizar la variación fustal de las tensiones de crecimiento longitudinales $\left(\sigma_{L}\right)$, del indicador de la tensión de crecimiento $(G S I)$ y del módulo de elasticidad de la madera en tensión $\left(\boldsymbol{E}_{T}\right)$, cada una de estas variables fueron obtenidas en cuatro posiciones distintas alrededor de la circunferencia del árbol (exposiciones Norte, Sur, Este y Oeste del fuste), a la altura fija de $2 \mathrm{~m}$, y al 33, 66 y 100\% de la altura comercial del árbol $(A C$, con un diámetro límite de utilización $=20 \mathrm{~cm}$ ), configurándose un total de 16 posiciones de muestreo distintas en el fuste. Las alturas relativas 33, 66 y $100 \%$ de la $\boldsymbol{A C}$, promedio de los 9 árboles, correspondieron a los 8,16 y 24 m respectivamente.

\section{Indicador de la tensión de crecimiento (GSI)}

Las mediciones del GSI fueron realizadas sobre el árbol abatido, en cada una de las 16 posiciones de muestreo configuradas, utilizando el método CIRAD-Forêt, basado en el método de un orificio único "the single hole method" (Fournier et al. 1994).

En la figura 1, se muestra el instrumento utilizado. Este está formado por dos clavos que se insertan en la madera (sin corteza), separados entre sí a una distancia de $45 \mathrm{~mm}$, a lo largo de la fibra, y además, una estructura metálica que contiene un comparador digital, y que es apoyada sobre los clavos una vez insertos en la madera. Para liberar las $\sigma_{L}$ en la periferia del fuste, se realiza un orificio con una broca de $20 \mathrm{~mm}$ de diámetro en el punto medio entre los dos clavos, y como consecuencia, estos clavos se separan. Este desplazamiento de los clavos es registrado por el comparador digital, y corresponde al GSI medido en micrómetros. 


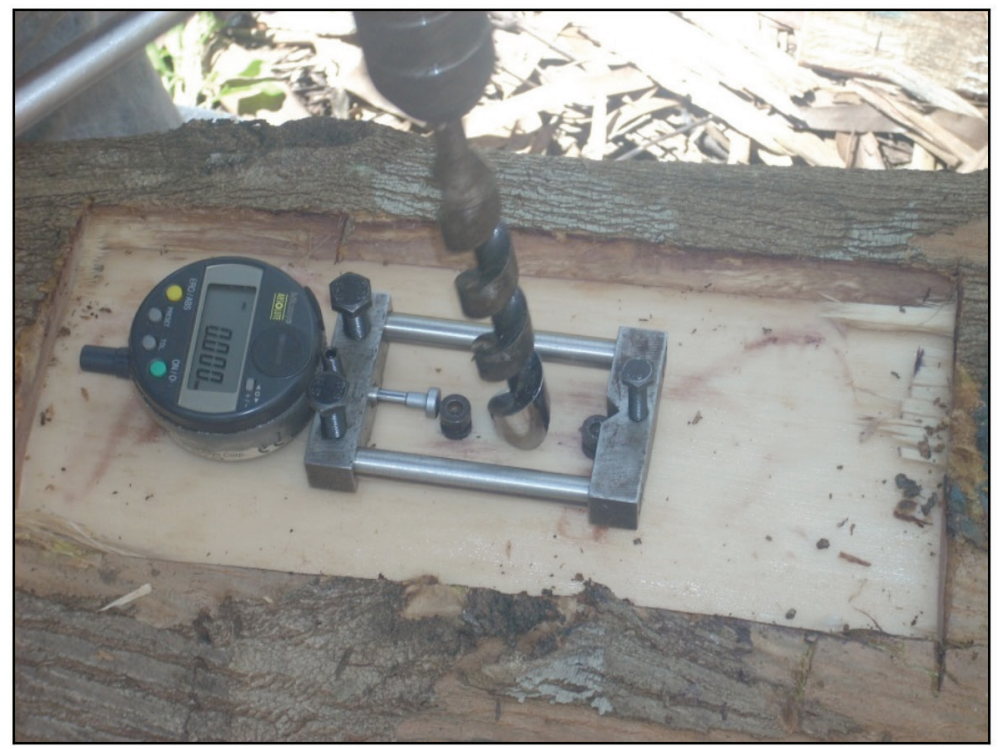

Figura 1. Instrumento para medir el indicador de la tensión de crecimiento, GSI, según el método CIRAD-Fôret.

\section{Módulo de elasticidad de la madera en tensión $\left(E_{T}\right)$}

Vigas de madera de $2 \times 2 \times 30 \mathrm{~cm}$, en alto, ancho y largo respectivamente, fueron extraídas de cada una de las 16 posiciones de muestreo configuradas, lo más cercano posible al punto de medición del GSI. En total se obtuvieron 144 vigas en estado verde, que fueron sometidas a flexión estática bajo una carga centrada en la cara tangencial, a una razón $l / h$ de 14 (donde $l$ y $h$ es la luz y el alto de la viga, respectivamente), en una máquina universal de ensayos.

Debido a que los valores de módulo de elasticidad obtenidos en los ensayos de flexión, corresponden a valores aparentes (MOEs aparentes, Bodig y Jayne 1982), estos debieron ser corregidos por los efectos del cizalle paralelo a la fibra. Para realizar estas correcciones, se condujeron ensayos anexos en flexión, para seis vigas de $2 \times 2 \times 55 \mathrm{~cm}$, en alto, ancho y largo respectivamente, las que fueron extraídas al azar de las distintas posiciones de los árboles muestreados. Estas vigas fueron ensayadas a distintas relaciones $l / h(8$, 14, 18, 22 y 24), siguiendo la metodología propuesta por Timoshenko (1955) y utilizado por Biblis (2001, 1965) y Adamopoulos et al. (2007), con el objetivo de obtener el módulo de elasticidad real, E, y el módulo de rigidez, G, en flexión.

De este ensayo se obtuvo un $E$ de $14010 \mathrm{MPa}$ y un $G$ de $350 \mathrm{MPa}$, donde la relación $E / G$ fue de 40 . Con este valor, se corrigieron los $M O E$ s aparentes de las 144 vigas, siguiendo las deducciones de Bodig y Jayne (1982), y se obtuvieron sus módulos de elasticidad reales en flexión, Es, sin efectos del cizalle, los cuales son equivalentes a los módulos de elasticidad en tensión $E_{T}$, paralelo a la fibra. En promedio los $M O E$ s aparentes de las vigas incrementaron en un $20 \%$.

\section{Tensiones de crecimiento longitudinales $\left(\sigma_{L}\right)$}

Las tensiones de crecimiento longitudinales fueron calculadas como el producto entre el $\boldsymbol{E}_{T}$ y la deformación de crecimiento longitudinal $\left(\varepsilon_{L}\right)$. Esta última, que fue obtenida a través de la relación, $\varepsilon_{L}=12,3^{-6} \mathrm{x} G S I$, que utiliza un factor de conversión aproximado para angiospermas estándar (Fournier et al. 1994). 


\section{Análisis de datos}

La variación fustal de las variables de interés $\left(\sigma_{L}, G S I\right.$ y $\left.E_{T}\right)$, fueron examinadas mediante el ajuste de modelos lineales mixtos, que tuvieron la siguiente estructura:

$$
Y_{i j k}=\mu+\alpha_{i}+\tau_{j}+b_{k}+(\alpha \tau)_{i j}+e_{i j k}
$$

donde $Y_{i j k}$ es la observación realizada sobre la $i$-ésima altura, en la $j$-ésima exposición del $k$-ésimo árbol, $\mu$ es la media total, $\alpha_{i}$ es el efecto fijo de la $i$-ésima altura (con $i=2 \mathrm{~m}, 33 \% A C, 66 \% A C$ y $A C$ ), $\tau_{j}$ es el efecto fijo de la $j$-ésima exposición (con $j=$ Norte, Sur, Este y Oeste), $(\alpha \tau)_{i j}$ es la interacción entre la $i$-ésima altura y la j-ésima exposición, $b_{k}$ es el efecto aleatorio del $k$-ésimo árbol y $e_{i j k}$ es el residual aleatorio.

Los modelos fueron ajustados utilizando el procedimiento PROC MIXED del software estadístico SAS (SAS 2009). La significancia estadística de los efectos fijos fue testeada mediante el valor $\boldsymbol{F}$ computado por el procedimiento, y los contrastes entre medias fueron realizados mediante la prueba HSD de Tukey. Las relaciones entre variables fueron realizadas a través de análisis de regresión lineal simple.

\section{RESULTADOS Y DISCUSIÓN}

\section{Niveles de tensiones de crecimiento longitudinales $\left(\sigma_{L}\right)$}

Los niveles de $\sigma_{L}$ obtenidos a distintas alturas y exposiciones del fuste se encontraron en un rango de 10,5 a 56,1 MPa; con una media global de 28,3 MPa y un coeficiente de variación $(C V)$ de $31,4 \%$. Una alta variabilidad ha sido reportada entre árboles de una misma especie, y también dentro de un mismo árbol, para los niveles de $\sigma_{L}$ (Nicholson 1973), y también para su estimador, el GSI (Raymond et al. 2004, Muneri et al. 1990). El amplio rango de $\sigma_{L}$ observados en este estudio, posiblemente refleja tales diferencias.

La magnitud promedio encontrada para las $\sigma_{L}$, es superior a la que reportan árboles de la misma especie a la edad de 8 años (Chafe 1990), y a la que reportan árboles adultos de Eucalyptus oblicua (Nicholson 1973) y Eucalyptus regnans (Nicholson 1973, Chafe 1990), sin embargo, es menor a la que exhiben árboles de Eucalyptus dunni a los 8,13,15 y 19 años de edad (Trugilho y Oliveira 2008) y similar a la que presentan clones de Eucalyptus a los 6 años (Trughilho et al. 2002). Aun así, se encuentra dentro del rango de valores publicados para distintas especies del género Eucalyptus. Aunque también, se debe considerar en las comparaciones más directas, que las metodologías empleadas para estimar las $\sigma_{L}$, difieren entre la mayoría de los estudios, al igual que las posiciones en el fuste en que estas $\sigma_{L}$ son muestreadas.

En el caso del GSI, obtenido a través de un método estándar "CIRAD-Fôret", es ampliamente utilizado para estimar el nivel de $\sigma_{L}$. Como referencia, los niveles del GSI encontrados en este estudio tuvieron un valor medio de $147 \mu \mathrm{m}$, con un $\boldsymbol{C V}$ de 26,1\%; y son similares a los que reportan árboles de la misma especie a la edad de 15 años, creciendo en la misma área de estudio (Díaz et al. 2012), pero son bastante mayores al GSI medio de $58 \mu \mathrm{m}$ (Medhurst et al. 2011) y $70 \mu \mathrm{m}$ (Valencia et al. 2011), que reportan árboles de Eucalyptus nitens de 22 años creciendo en Australia. No obstante, en estos estudios el GSI fue medido sólo a 1,3 m sobre el nivel del suelo.

\section{Variación fustal de las tensiones de crecimiento longitudinales, indicador de la tensión de crecimiento y módulo de elasticidad de la madera en tensión.}

Los resultados del análisis de los efectos fijos (Tabla 1), indicaron que la altura en el fuste tuvo un efecto altamente significativo sobre las $\sigma_{L}$, el GSI y el $E_{T}$. Para el caso de la exposición en el fuste, su efecto fue altamente significativo en las $\sigma_{L}$ y el $G S I$, y significativo en el $E_{T}$. La interacción altura-exposición no tuvo un efecto en las $\sigma_{L}, G S I$ y $E_{T}$. 
Tabla 1. Significancia de los efectos: altura y exposición en el fuste, y de su interacción, en las tensiones de crecimiento longitudinales $\left(\sigma_{L}\right)$, indicador de la tensión de crecimiento $(G S I)$ y módulo de elasticidad de la madera en tensión $\left(E_{m}\right)$.

\begin{tabular}{|lllllll|}
\hline \multirow{2}{*}{ Efectos } & \multicolumn{2}{c}{$\sigma_{L}$} & \multicolumn{2}{c}{$G S I$} & \multicolumn{2}{c|}{$E_{T}$} \\
\cline { 2 - 7 } & F-Valor & $\mathbf{P r}>\mathbf{F}$ & F-Valor & $\mathbf{P r}>\mathbf{F}$ & F-Valor & $\mathbf{P r}>\mathbf{F}$ \\
\hline Altura & 17,27 & $<0,001$ & 9,05 & $<0,001$ & 15,55 & $<0,001$ \\
Exposición & 11,22 & $<0,001$ & 10,57 & $<0,001$ & 3,28 & $<0,05$ \\
Altura*Exposición & 0,57 & 0,8170 & 0,77 & 0,6475 & 0,80 & 0,6170 \\
\hline
\end{tabular}

El efecto individual de la altura en el fuste, sobre las $\sigma_{L}$, el GSI y el $E_{T}$, se presenta en la Tabla 2. Los niveles medios de $\sigma_{L}$ encontrados al $33 \%$ de la $A C(34,8 \mathrm{MPa})$, fueron significativamente mayores a los encontrados en las otras alturas de muestreo $(P<0,05)$. De esta misma posición ( $33 \%$ de la $A C)$, la magnitud de las $\sigma_{L}$ disminuyó de forma significativa con la altura, hasta la $A C$. Los mayores niveles del GSI, también fueron encontrados al $33 \%$ de la $A C$ siendo significativo respecto al resto $(P<0,05)$, sin embargo, no se reportaron diferencias entre las otras alturas. En el caso del $E_{T}$, sus valores más altos fueron encontrados al 33 y $66 \%$ de la $A C$, y fueron significativamente mayores a los encontrados en la altura de $2 \mathrm{~m}(\mathrm{P}<0,05)$.

Tabla 2. Efecto de la altura en el fuste sobre las tensiones de crecimiento longitudinales $\left(\sigma_{L}\right)$, indicador de la tensión de crecimiento $(G S I)$ y módulo de elasticidad de la madera en tensión $\left(E_{T}\right)$, junto con los valores medios obtenidos por altura.

\begin{tabular}{|llll|}
\hline Altura & $\sigma_{L}(\mathbf{M P a})$ & $\boldsymbol{G S I}(\boldsymbol{\mu m})$ & $\boldsymbol{E}_{\boldsymbol{T}}(\mathbf{M P a})$ \\
\hline $2 \mathrm{~m}$ & $26,0 \mathrm{bc}$ & $147 \mathrm{~b}$ & $14366,1 \mathrm{c}$ \\
$33 \% \mathrm{AC}$ & $34,8 \mathrm{a}$ & $167 \mathrm{a}$ & $16819,9 \mathrm{a}$ \\
$66 \% \mathrm{AC}$ & $28,4 \mathrm{~b}$ & $144 \mathrm{~b}$ & $16011,1 \mathrm{ab}$ \\
$\mathrm{AC}$ & $24,1 \mathrm{c}$ & $129 \mathrm{~b}$ & $15204,3 \mathrm{bc}$ \\
\hline
\end{tabular}

NOTA: Promedios seguidos de letras iguales en la misma columna no difieren significativamente a $\boldsymbol{P}<0,05$.

En general, los niveles medios de las $\sigma_{L}$, del GSI y del $E_{T}$, tendieron a aumentar desde la base del fuste (altura de $2 \mathrm{~m}$ ) hasta el $33 \%$ de la $A C$, y desde esta misma posición, disminuyeron con la altura, hasta la $A C$. Los patrones de variación axial en el fuste, para las $\sigma_{L}$, el GSI y el $E_{T}$, aún no han sido bien entendidos, especialmente en árboles adultos de Eucalyptus. Aun así, el aumento en los niveles del GSI encontrado en este estudio, entre los $2 \mathrm{~m}$ y el $33 \%$ de $A C(8 \mathrm{~m})$, concuerda con el que reportan árboles de Eucalyptus globulus, a los 20 y 23 años de edad, entre los 1,3 y 3,7 m (Raymond et al. 2004).

Árboles jóvenes de Eucalyptus nitens, a la edad de 8 años, reportan una disminución en los niveles de tensiones de crecimiento, módulo de elasticidad de la madera y deformación longitudinal, con la altura en el fuste, entre los 1,5 y $15 \mathrm{~m}$ (Chafe 1985). En el presente estudio, las $\sigma_{L}$, el $\boldsymbol{G S I}$ y el $\boldsymbol{E}_{T}$, mostraron un patrón similar, pero entre los 8 y $24 \mathrm{~m}$. Al parecer, tanto en árboles jóvenes como en árboles adultos de Eucalyptus nitens, estas variables mecánicas disminuyen con la altura en el fuste. Sin embargo, la altura axial para los mayores niveles de estas variables mecánicas, parece diferir con la edad de los individuos. 
El efecto individual de la exposición en el fuste, sobre las $\sigma_{L}$, el GSI y el $E_{T}$, se presenta en la tabla 3. Los niveles de $\sigma_{L}$ encontrados en la exposición Sur del fuste, fueron estadísticamente mayores $(\mathrm{P}<0,05)$ a los encontrados en la exposiciones Norte y Oeste. En el caso del GSI, solo los valores encontrados en la exposición Norte fueron distintos, y significativamente menores $(P<0,05)$. Los valores de $E_{T}$ observados en la exposición Sur, fueron mayores y diferentes estadísticamente a los observados en la exposición Oeste $(P<0,05)$.

Tabla 3. Efecto de la exposición en el fuste sobre las tensiones de crecimiento longitudinales $\left(\sigma_{L}\right)$, indicador de la tensión de crecimiento $(G S I)$ y módulo de elasticidad de la madera $\left(E_{T}\right)$, junto con loc valores medins ohtenidos nor exnncición

\begin{tabular}{|llll|}
\hline Exposición & $\sigma_{L}(\mathbf{M P a})$ & $\boldsymbol{G S I}(\boldsymbol{\mu m})$ & $\boldsymbol{E}_{\boldsymbol{T}}$ (MPa) \\
\hline Sur & $32 \mathrm{a}$ & $158 \mathrm{a}$ & $16324,7 \mathrm{a}$ \\
Este & $30,6 \mathrm{ab}$ & $161 \mathrm{a}$ & $15369,6 \mathrm{ab}$ \\
Oeste & $27,0 \mathrm{bc}$ & $143 \mathrm{a}$ & $15307,5 \mathrm{~b}$ \\
Norte & $23,7 \mathrm{c}$ & $124 \mathrm{~b}$ & $15399,3 \mathrm{ab}$ \\
\hline
\end{tabular}

NOTA: Promedios seguidos de letras iguales en la misma columna no difieren significativamente a $\boldsymbol{P}<0,05$.

Variaciones en los niveles de $\sigma_{L}$ con la exposición en el fuste, reportadas en árboles adultos de Eucalyptus regnans y Eucalyptus oblicua, son principalmente asociadas a los requerimientos de soporte del árbol (Nicholson 1973), y además, los altos niveles de tensiones de crecimiento, son asociados a la presencia de madera de reacción (Yang y Waugh 2001). Diferencias en los niveles de GSI entre exposiciones, también han sido reportadas en árboles de Eucalyptus nitens (Valencia et al. 2011, Díaz et al. 2012) y Eucalyptus globulus (Raymond et al. 2004, Vignote et al. 1996), y los mayores niveles de GSI, reportados en ciertas exposiciones del fuste, son principalmente atribuidos a los efectos del viento predominante (Vignote et al. 1996, Medhurst et al. 2011, Valencia et al. 2011, Díaz et al. 2012).

En árboles de Eucalyptus nitens de 15 años de edad, crecidos en la misma área que los árboles utilizados en este estudio, se reportan diferencias significativas en los niveles de GSI y módulo de elasticidad dinámico de la madera, entre las exposiciones Norte y Sur del fuste, los cuales fueron siempre mayores en la exposición Sur (barlovento), frente a los vientos predominantes del lugar (Díaz et al. 2012). En el presente estudio, los niveles de $\sigma_{L}, G S I$ y $E_{T}$ encontrados en la exposición Sur, también fueron mayores a los encontrados en la exposición Norte del fuste. En efecto, las demandas mecánicas producidas por los vientos predominantes, pueden ser sugeridas como una de las posibles causas de las variaciones encontradas.

Aunque los árboles estudiados se encontraban aparentemente rectos, éstos presentaron una alta variabilidad dentro del fuste, en los niveles de $\sigma_{L}$, GSI y $E_{T}$. Sin embargo, también se ha señalado que en fustes verticales, las $\sigma_{L}$ son desarrolladas como una adaptación a las demandas mecánicas sobre el fuste (Gartner 1997), como una respuesta o en "anticipación" a éstas.

De acuerdo con los resultados encontrados en este estudio, los mayores niveles de tensiones de crecimiento longitudinales se encontraron al $33 \%$ de la $A C$ del árbol, así como también, estas tensiones fueron significativamente mayores en la exposición Sur a lo largo del fuste aserrable. Por lo tanto, se espera que en esta altura y exposición a lo largo del fuste, se presenten los mayores defectos asociados a las altas tensiones de crecimiento. 


\section{Relaciones entre las tensiones de crecimiento longitudinales, indicador de la tensión de crecimiento y módulo de elasticidad de la madera en tensión}

La relación encontrada entre el $\boldsymbol{G S} \boldsymbol{I}$ y el $\boldsymbol{E}_{T}$, aunque fue significativa $(\boldsymbol{P}<0,05)$, fue bastante débil $\left(\mathrm{R}^{2}=\right.$ 0,035; Figura 2a). Relaciones similares han sido reportadas en árboles de E. globulus (Yang y Ilic 2003), y en árboles de Eucalyptus nitens y Eucalyptus regnans (Chafe 1990), pero entre el módulo de elasticidad de la madera y la deformación longitudinal, sin embargo, en ninguno de los casos esta relación fue significativa.

La relación encontrada entre las $\sigma_{L}$ y el GSI fue fuerte y significativa $(P<0,01)$, con un coeficiente de correlación igual a 0,91 (Figura $2 \mathrm{~b}$ ). Esta fuerte correlación encontrada entre el $\sigma_{L}$ y el GSI, es similar a la que reportan árboles de Eucalyptus dunnii a las edades de 13, 15 y 19 años (Trugilho y Oliveira 2008), y también similar a la relación entre el $\sigma_{L}$ y la deformación longitudinal que reportan árboles de Eucalyptus globulus de 10 años (Yang y Ilic 2003) y árboles de Eucalyptus nitens y Eucalyptus regnans de 8 y 36 años respectivamente (Chafe 1990).
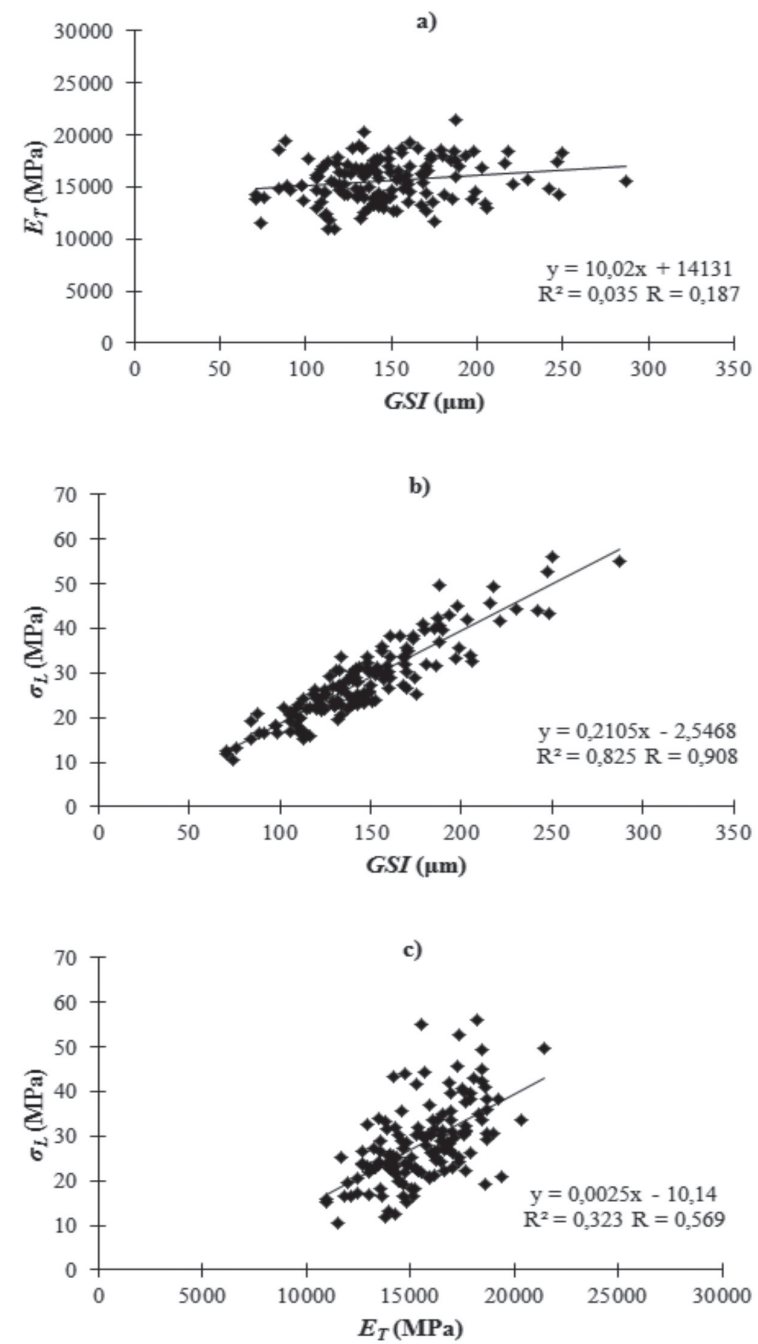

Figura 2. Relaciones entre las tensiones de crecimiento longitudinales $\left(\sigma_{L}\right)$, indicador de la tensión de crecimiento $(G S I)$ y módulo de elasticidad de la madera en tensión $\left(E_{T}\right)$. a) $E_{T}$ versus $G S I$, b) $\sigma_{L}$ versus 
La relación entre las $\sigma_{L}$ y el $E_{T}$ fue significativa $(P<0,01)$ y positiva $(\mathrm{R}=0,57$; Figura 3$)$, pero más débil a la relación encontrada entre las $\sigma_{L}$ y el GSI. En árboles de la misma especie, a la edad 10 años, se han reportado relaciones similares pero poco consistentes (Chauhan y Walker 2004).

Aunque los valores de $E_{T}$ y $G S I$ están involucrados en el cálculo de las tensiones de crecimiento, la contribución efectiva de cada uno de ellos, puede ser examinada a través de su relación con los niveles de $\sigma_{L}$ (Chafe 1990). Como se muestra en la Figura 2b, la relación entre las $\sigma_{L}$ y el GSI fue más fuerte y significativa que la relación entre las $\sigma_{L}$ y el $E_{T}$ (Figura 2c), y de acuerdo con Trugilho y Oliveira (2008), estos resultados indican que la influencia del GSI, en la estimación de los niveles de $\sigma_{L}$, es mayor a la del $E_{T}$, por lo tanto, el GSI puede ser utilizado como un buen estimador de las tensiones de crecimiento.

\section{CONCLUSIONES}

Los niveles de tensiones de crecimiento longitudinales $\left(\sigma_{L}\right)$, encontrados en árboles de $E$. nitens con dimensiones aserrables, estuvieron en un rango de 10,5 a 56,1 MPa, y en general, se encuentran dentro del rango de valores publicados para especies del género Eucalyptus.

Las tensiones de crecimiento longitudinales $\left(\sigma_{L}\right)$, el indicador de la tensión de crecimiento $(G S I)$ y el módulo de elasticidad de la madera $\left(E_{T}\right)$, variaron significativamente a través de las alturas y exposiciones en el fuste.

Los mayores niveles de tensiones de crecimiento longitudinales $\left(\sigma_{L}\right)$ se encontraron al $33 \%$ de la $A C$ del árbol. Y estas tensiones fueron significativamente mayores en la exposición Sur a lo largo del fuste aserrable.

El indicador de la tensión de crecimiento, GSI, puede ser utilizado como un buen estimador de las tensiones de crecimiento $\left(\sigma_{L}\right)$.

\section{AGRADECIMIENTOS}

Los autores hacen expreso su agradecimiento a la Empresa Forestal Mininco S.A., por facilitar el material para el desarrollo de este estudio. 


\section{BIBLIOGRAFIA}

Adamopoulos, S.; Passialis, C.; Voulgaridis, E. 2007. Strength properties of juvenile and mature wood in black locust (Robinia pseudoacacia L.). Wood and Fiber Science 39 (2): 241-249.

Baillères, H. 1994. Précontraintes de croissance et propriétés mécano-physiques de clones d'Eucalyptus (Pointe Noire-Congo): hétérogénéités, corrélations et interprétations histologiques. L'Université de Bordeaux I, 162p.

Biblis, E.J. 1965. Analysis of wood-fiberglass composite beams within and beyond the elastic region. Forest Products Journal 15 (2): 81-88.

Biblis, E.J. 2001. Tension parallel to grain, pure flexural stiffness, and modulus of rigidity of clear wood of seven eastern read oaks. Forest Products Journal 51 (4): 87-89.

Biechele, T.; Nutto, L.; Becker, G. 2009. Growth strain in Eucalyptus nitens at different stages of development. Silva Fennica 43 (4): 669-679.

Bodig, J.; Jayne, A. 1982. Mechanics of wood and wood composites. Van Nostrand Reinhold Company Inc. $712 \mathrm{p}$.

Chafe, S.C. 1985. Variation in longitudinal growth stress with height in trees of Eucalyptus nitens Maiden. Australian Forest Research 15 (1): 51-55.

Chafe, S.C. 1990. Relationships among growth strain, density and strength properties in two species of Eucalyptus. Holzforschung 44 (6): 431-437.

Chauhan, S.; Walker, J. 2004. Relationships between longitudinal growth strain and some wood properties in Eucalyptus nitens. Australian Forestry 67 (4): 254-260.

Díaz, S.; Espinoza, M.; Valenzuela, L.; Cancino, J.; Lasserre, J.P. 2012. Efecto del raleo en el crecimiento y algunas propiedades de la madera de Eucalyptus nitens en una plantación de 15 años. Maderas. Ciencia y tecnología 14 (3): 373-388.

Fournier, M.; Chanson, B.; Thibaut, B.; Guitard, D. 1994. Mesures des déformations résiduelles de croissance à la surface des arbres, en relation avec leur morphologie. Observations sur différentes espèces. Annals of Forest Science 51: 249-266.

Gartner, B.L. 1997. Trees have higher longitudinal growth strains in their stems than in their roots. Journal Plant Science 158 (4): 418-423.

INFOR. 2004. Eucalyptus nitens en Chile: Primera monografía. Informe Técnico $\mathrm{N}^{\circ} 165$. Valdivia, Chile. Instituto Forestal. 143p.

Malan, F.S.; Gerischer, G.F.R. 1987. Wood property differences in South African grown Eucalyptus grandis trees of different growth stress levels. Holzforschung 41 (6): 331-335. 
Medhurst, J.; Ottenschlaeger, M.; Wood, M.; Harwood, C.; Beadle, C; Valencia, J.C. 2011. Stem eccentricity, crown dry mass distribution, and longitudinal growth strain of plantation-grown Eucalyptus nitens after thinning. Canadian Journal of Forest Research 41: 2209-2218.

Muneri, A.; Leggate, W.; Palmer, G. 1999. Relationships between surface growth strain and some tree, wood and sawn timber characteristics of Eucalyptus cloeziana. Southern African Forestry Journal 186: 41-49.

Nicholson, J.E. 1973. Growth stress differences in eucalypts. Forest Science 19: 169-174.

Raymond, C.A.; Kube, P.D.; Pinkard, L.; Savage, L.; Bradley, A.D. 2004. Evaluation of non-destructive methods of measuring growth stress in Eucalyptus globulus: relationships between strain, wood properties and stress. Forest Ecology and Management 190: 187-200.

SAS Institute inc. 2009. SAS/STAT ${ }^{\circledR} 9.2$ user's guide, second edition. Cary, NC: SAS Institute Inc.

Timoshenko, S. 1955. Strenght of materials. 3rd. Ed. Part I. Elementary theory and problems. Van Nostrand, New York.

Touza, M.C. 2001. Tensiones de crecimiento en Eucalyptus globulus de Galicia (España). Influencia de la silvicultura y estrategias de aserrado. Maderas. Ciencia y tecnología 3 (1-2): 68-89.

Trugilho, P.F.; Lima, J.T.; Rosado, S.C.S.; Mendes, L.M.; Mori, F.A.; Souza, M.A.M. 2002. Avaliação da tensão de crescimento em clones de Eucalyptus. Floresta e Ambiente 9 (1): 38-44.

Trugilho, P.F.; Lima, J.T.; de Pádua, F.A.; de Carvalho, L.; Andrade C.R. 2006. Deformação residual longitudinal (DRL) e tangencial (DRT) em seis clones de Eucalyptus spp. Cerne 12 (3): 279-286.

Trugilho, P.F.; Oliveira, J.T. 2008. Relationships and estimates of longitudinal growth stress in Eucalyptus dunnii at different ages. Revista Árbore 32 (4): 723-729.

Valdés, R. 2004. Determinación de tensiones de crecimiento de Eucalyptus nitens (Deane \& Maiden) Maiden mediante un método no destructivo. Memoria de título para optar al grado de ingeniero en industrias de la madera, Universidad de Talca, Chile. 48p.

Valencia, J.C.; Cabrera, J.A. 2009. Modelo de consorciamiento ciencia-empresa orientado a rentabilizar plantaciones de Eucalyptus nitens en Chile. ECOGESTION 2009. Primera reunión sobre planificación y legislación forestal. p. 42-49.

Valencia, J.; Hardwood, C.; Washusen, R.; Morrow, A.; Wood, M.; Volker, P. 2011. Longitudinal growth strain as a log and wood quality predictor for plantation-grown Eucalyptus nitens sawlogs. Wood Science and Technology 45: 15-34.

Vignote, S.; Molinero, I.; Gerard, J.; Diez, M.R. 1996. Estudio de las tensiones de crecimiento de Eucalyptus globulus Labill en Galicia y su relación con las características de estación y morfológicas del propio árbol. Investigación Agraria. Sistemas y Recursos Forestales 5 (1): 153-165. 
Yang, J.L.; Fife, D.; Matheson, A.C. 2001. Growth strain in three provenances of plantation-grown Eucalyptus globulus Labill. Australian Forestry 64 (4): 248-256.

Yang, J.L.; Waugh, G. 2001. Growth stress, its measurement and effects. Australian Forestry 64 (2): 127-135.

Yang, J.L.; Ilic, J. 2003. A new method of determining growth stress and relationships between associated wood properties of Eucalyptus globulus Labill. Australian Forestry 66 (2): 153-157.

Yang, J.L.; Baillères, H.; Okuyama, T.; Muneri, A.; Downes, G. 2005. Measurement methods for longitudinal surface strain in trees: a review. Australian Forestry 68 (1): 34-43. 